\title{
Value of the preoperative prognostic nutritional index for the evaluation of patient prognosis after radical gastrectomy
}

\author{
LILI WANG, YONGZHI MIAO, TIANWEN CHEN, DENGZHONG SUN, SITANG GE, \\ LUGEN ZUO and MULIN LIU \\ Department of Gastrointestinal Surgery, The First Affiliated Hospital of Bengbu Medical College, \\ Bengbu, Anhui 233004, P.R. China
}

Received July 21, 2019; Accepted December 6, 2019

DOI: $10.3892 / \mathrm{mco} .2020 .1980$

\begin{abstract}
Preoperative prognostic nutritional index (PNI) has been widely used for the clinical evaluation of patients with cancer. The present study assessed the prognostic value of preoperative PNI in patients after gastric cancer (GC) radical surgery. The clinical case and follow-up data of 170 patients undergoing GC radical surgery were retrospectively analyzed. The receiver operating characteristic (ROC) curve was used to compare the predictive ability of each inflammatory index: The PNI, neutrophil-to-lymphocyte ratio (NLR) and lymphocyte-to-monocyte ratio (LMR). The correlation between the preoperative PNI and overall survival (OS) was also analyzed via Kaplan-Meier (K-M) curves and multivariate Cox regression analyses. The results revealed that the optimal PNI cut-off was 46.030. According to this cut-off value, the whole sample was divided into PNI <46.030 (low PNI group) and PNI $\geq 46.030$ (high PNI group). These groups were comprised of 102 and 68 cases, respectively. The area under the curve value of the PNI was 0.725 , which was greater than that of traditional inflammatory indices, including the NLR and LMR. K-M survival analysis revealed that the 5 year survival rate of patients in the low PNI group was significantly lower than that of patients in the high PNI group $(\mathrm{P}<0.01)$. Univariate analysis and Cox multiple regression model analysis demonstrated that the $\mathrm{T}$ stage, $\mathrm{N}$ stage, pathological grade and PNI were independent risk factors for the 5 year survival rate after radical gastrectomy $(\mathrm{P}<0.05)$. In conclusion, the preoperative
\end{abstract}

Correspondence to: Dr Mulin Liu, Department of Gastrointestinal Surgery, The First Affiliated Hospital of Bengbu Medical College, 287 Changhuai Road, Bengbu, Anhui 233004, P.R. China

E-mail: liumulin66@aliyun.com

Abbreviations: PNI, prognostic nutritional index; NLR, neutrophilto-lymphocyte ratio; LMR, lymphocyte-to-monocyte ratio; AUC, area under the curve; MST, median survival time; CI, confidence interval

Key words: gastric cancer, prognostic nutritional index, neutrophil-to-lymphocyte ratio, lymphocyte-to-monocyte ratio, survival analysis, risk factor analysis
PNI is an independent risk factor for 5 year survival after radical gastrectomy and has clinical value for the prognostic evaluation of patients with GC.

\section{Introduction}

Gastric cancer (GC) is one of the most common malignant tumors of the digestive system. The 2018 Global Cancer Report shows that more than half of the 18.1 million new cancer cases and 9.6 million cancer-related deaths worldwide occur in Asia. GC ranks as the sixth most common newly diagnosed cancer, with an incidence of $5.7 \%$; however, the mortality rate is as high as $8.2 \%$, second only to mortality due to lung cancer (1). Although comprehensive treatment based on surgery in recent years has further improved the treatment of patients with GC, the overall prognosis of patients with GC is still poor $(2,3)$. Therefore, it is particularly important to effectively predict the survival of patients with GC and to develop individualized treatment options. In the past, combined with the comprehensive evaluation of intraoperative and postoperative pathology, tumor biomarkers, such as the pathological stage, depth of invasion, number of involved lymph nodes, and presence or absence of surrounding tissues, are the key factors used to determined the prognosis of patients (4). However, the prognosis of patients with GC is related not only to tumor biomarkers but also to the body's inflammation and immunity.

In recent years, the importance of preoperative nutritional status and immunity in patients with GC has been recognized $(5,6)$. The preoperative nutritional status and immunity of patients with GC determine whether the patient can undergo surgery and are some of the important factors in the evaluation of postoperative complications, postoperative recurrence and metastasis, chemotherapy tolerance and long-term prognosis (7-10). Li et al (11) showed that the implementation of effective preoperative nutrition and immune supportive treatments can improve the quality of life and long-term prognosis of patients with GC. The prognostic nutritional index (PNI) is a simple and convenient indicator for assessing preoperative nutritional status and immunity in patients (12). In recent years, it has been used for the clinical evaluation of prognosis in patients with a variety of cancers. However, few studies have explored the value of the preoperative PNI compared with the value of traditional inflammatory markers in the prognosis 
of patients after GC radical surgery. This study investigated the value of the preoperative PNI compared with the value of traditional inflammatory indicators in the prognostic evaluation of patients undergoing GC radical surgery to provide a reference for clinical diagnosis and treatment.

\section{Patients and methods}

Patients. We retrospectively assessed all patients with resectable GC who were treated between January 1, 2010, and January 15, 2013, at the Department of Gastrointestinal Surgery of The First Affiliated Hospital of Bengbu Medical College (Bengbu, China). The following inclusion criteria were applied: i) GC was preoperatively confirmed by pathology and histopathology; ii) patient had not undergone neoadjuvant radiotherapy and chemotherapy before surgery; iii) had no infectious diseases before blood collection; iv) was confirmed by imaging to have no distant metastasis; v) patient underwent radical gastrectomy; vi) follow-up occurred during or after March, and vii) patient died of GC or gastric cancer-related diseases. The following exclusion criteria were applied: i) Patient could not tolerate surgery due to severe liver and kidney dysfunction; ii) had an active infection; iii) had autoimmune disease; iv) had other malignant tumors and end-stage diseases, and v) patient was missing clinical data. Finally, 170 patients were included in the study. Clinical data, such as sex, age, recent weight loss, histological type, tumor location, tumor size, TNM stage, and pathological stage, were collected. The results of the first blood collection of patients admitted to the hospital included neutrophil count, lymphocyte count, monocyte count, and serum albumin concentration. The NLR, LMR, and PNI were calculated. The NLR was calculated as the neutrophil count divided by the lymphocyte count. The LMR was calculated as the lymphocyte count divided by the monocyte count. The PNI was equal to the serum albumin $(\mathrm{g} / \mathrm{L})+5^{*}$ lymphocyte absolute count $\left(10^{9} / \mathrm{L}\right)(12)$. Surgical procedures were performed according to the Japanese Gastric Cancer Treatment Guidelines (13). The pathological staging of all enrolled patients was performed according to the eighth edition of the AJCC guidelines for histopathological findings (14). This study was approved by the Ethics Committee of the First Affiliated Hospital of Bengbu Medical College. All patients provided signed informed consent.

Follow-up. All patients were followed up with a regular clinical visit and by telephone. A postoperative follow-up assessment was performed every 3 months for 5 years and then every 6 months during years $3-5$. Follow-up included a physical examination; laboratory and imaging examinations; laboratory tests, including routine blood tests, routine biochemical tests, and tumor markers; and imaging examinations, including a chest X-ray and an enhanced abdominal CT examination. The clinical follow-up time was from the date of surgery to the time of death or the deadline, which was December 2018.

Statistical analysis. Data analysis was performed using SPSS Statistics 23.0 software (ver. 23.0; IBM Corp.). Measurement data were expressed as mean \pm SD. Differences between groups were analyzed by one-way analysis of variance (ANOVA) and Student's t-tests. The $\chi^{2}$ test was used to compare the count

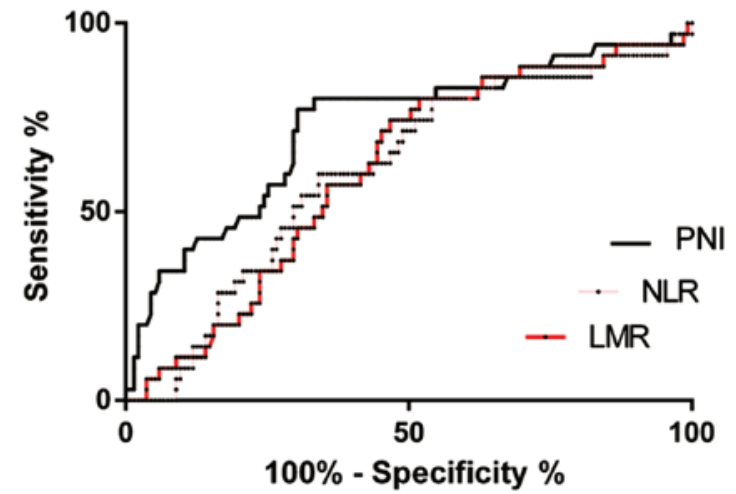

Figure 1. The value of various inflammatory markers in the survival of patients after GC radical resection. The area under the curve of PNI is significantly higher than that of NLR and LMR. PNI, prognostic nutritional index; NLR, neutrophil-to-lymphocyte ratio; LMR, lymphocyte-to-monocyte ratio.

data, and Fisher's exact test was used when the sample size was $<5$. The diagnostic value of each inflammatory index for cancer-related death 5 years after radical gastrectomy was analyzed by receiver operating characteristic (ROC) curve analysis. The areas under the curves and the $\mathrm{Z}$ values were compared to determine the discriminatory ability of each inflammatory index. The overall survival (OS) time was the number of days from the day of surgery until the day of death. The survival rate was expressed by the Kaplan-Meier curve, and the log-rank $\chi^{2}$ test was used for comparison between the groups. Multivariate analysis of the 5 year survival rate after GC surgery was performed with the Cox proportional hazard regression model. All statistical tests were bilateral, and the significance was set to $\mathrm{P}<0.05$.

\section{Results}

The ROC curve was used to determine the cut-off value. The areas under the PNI, NLR, and LMR curves were $0.725,0.609$ and 0.609 , respectively. When the PNI was 46.030, the Youden index was at the maximum, with a sensitivity of $77.14 \%$ and a specificity of $69.69 \%$. When the NLR was 2.464 , the Youden index was at the maximum, with a sensitivity of $80.00 \%$ and a specificity of $45.93 \%$. When the LMR was 3.279 , the Youden index was at the maximum, with a sensitivity of $80.00 \%$ and a specificity of $48.15 \%$. In addition, the ROC curve showed that the area under the PNI curve was 0.725 , which was significantly higher than those of the NLR and LMR $(\mathrm{P}<0.05)$ (Fig. 1 and Table I).

Clinicopathological characteristics. The general clinical features of 170 patients with GC are shown in Table II. There were 127 males and 43 females. The average age of the patients was $61.14 \pm 11.47$ years old. The most common pathological type of tumor was adenocarcinoma, and the most common tumor site was the cardia. With regard to TNM staging, there were 15 patients with T1-T2 tumors, 155 patients with T3-T4 tumors, 77 patients with N0-N1 tumors, 93 patients with N2-N3 tumors, 53 patients with phase I/II tumors, and 117 patients with phase III tumors. PNI was significantly associated with sex, histological type, tumor size, $\mathrm{N}$ stage, pathological stage, the NLR, and the LMR $(\mathrm{P}<0.05)$ (Table II). 
Table I. Comparison of the receiver operating curves of inflammation indicators.

\begin{tabular}{lccccccc}
\hline Parameter & AUC & $95 \%$ CI & Cut-off & Sensitivity (\%) & Specificity (\%) & Z-value & P-value \\
\hline PNI & 0.725 & $0.623-0.826$ & 46.03 & 77.14 & 69.69 & - \\
NLR & 0.609 & $0.507-0.712$ & 2.464 & 80.00 & 45.93 & 2.228 \\
LMR & 0.609 & $0.510-0.708$ & 3.279 & 80.00 & 48.15 & 2.714 & $0.026^{\mathrm{a}}$ \\
\hline
\end{tabular}

${ }^{a} \mathrm{P}<0.05$. PNI, prognostic nutritional index; NLR, neutrophil-to-lymphocyte ratio; LMR, lymphocyte-to-monocyte ratio; AUC, area under the curve; CI, confidence interval.

Table II. General clinical characteristics of patients with gastric cancer.

Parameter $\quad$ PNI $<46.03(n=102) \quad$ PNI $\geq 46.03(n=68) \quad \chi^{2} / F \quad P-v a l u e$

Sex

Male

Female

Age (years)

$<60$

$\geq 60$

Recent weight loss (kg)

$<5$

$\geq 5$

Histological type

Adenocarcinoma

Other

Tumor location

Gastric cardia

Gastric body

Gastric antrum

Tumor size $(\mathrm{cm})$

$<5$

$\geq 5$

T stage

T1-T2

T3-T4

$\mathrm{N}$ stage

N0-N1

N2-N3

Pathological stage

I-II

III

NLR

$$
<2.464
$$$$
\geq 2.464
$$

LMR

$$
\begin{aligned}
& <3.279 \\
& \geq 3.279
\end{aligned}
$$

35

67

63

39

82

20

82

20

7

39

82

44

27

31

43

59

\section{6}

96

33

69

20

82

40

62

63

39
45

23

28

40

0.8238

$0.3641^{\mathrm{a}}$

46

22

0.6136

$0.4334^{\mathrm{a}}$

63

5

4.8851

$0.0271^{\mathrm{a}}$

22

18

28

2.5892

$0.2740^{\mathrm{b}}$

41

27

5.3691

$0.0205^{\mathrm{a}}$

9

59

2.7421

$0.0977^{\mathrm{a}}$

44

24

17.241

$<0.0001^{\mathrm{a}}$

33

35

15.911

$<0.0001^{\mathrm{a}}$

61

7

43.131

$<0.0001^{\mathrm{a}}$

9

59

12.581

$0.0004^{\mathrm{a}}$

P-values were determined using a ${ }^{a} \chi^{2}$ test and ${ }^{\mathrm{b}}$ ANOVA. PNI, prognostic nutritional index; NLR, neutrophil-to-lymphocyte ratio; LMR, lymphocyte-to-monocyte ratio. 
Table III. Univariate analysis and multivariate analysis of the 5-year survival of patients after radical gastrectomy.

\begin{tabular}{|c|c|c|c|c|c|c|}
\hline \multirow[b]{2}{*}{ Parameter } & \multicolumn{3}{|c|}{ Univariate analysis } & \multicolumn{3}{|c|}{ Multivariate analysis } \\
\hline & $\mathrm{n}$ & MST (months) & P-value & HR & $95 \% \mathrm{CI}$ & ${ }^{\text {ap}} \mathrm{P}$-value \\
\hline \multicolumn{7}{|l|}{ Sex } \\
\hline Male & 127 & 16.3 & & & & \\
\hline Female & 43 & 17.6 & 0.728 & - & - & - \\
\hline \multicolumn{7}{|l|}{ Age (years) } \\
\hline$<60$ & 63 & 17.5 & & & & \\
\hline$\geq 60$ & 107 & 15.9 & 0.204 & - & - & - \\
\hline \multicolumn{7}{|c|}{ Recent weight loss (kg) } \\
\hline$<5$ & 109 & 17.4 & & & & \\
\hline$\geq 5$ & 61 & 15.6 & 0.214 & - & - & - \\
\hline \multicolumn{7}{|l|}{ Histological type } \\
\hline Adenocarcinoma & 145 & 17.1 & & & & \\
\hline Other & 25 & 15.2 & 0.136 & - & - & - \\
\hline \multicolumn{7}{|l|}{ Tumor location } \\
\hline Gastric cardia & 66 & 15.75 & & & & \\
\hline Gastric body & 45 & 14.7 & & & & \\
\hline Gastric antrum & 59 & 27.4 & 0.007 & 0.821 & $(0.663-1.016)$ & 0.070 \\
\hline \multicolumn{7}{|l|}{ Tumor size $(\mathrm{cm})$} \\
\hline$<5$ & 84 & 20.45 & & & & \\
\hline$\geq 5$ & 86 & 14.75 & $<0.001$ & 1.026 & $(0.705-1.494)$ & 0.892 \\
\hline \multicolumn{7}{|l|}{ T stage } \\
\hline $\mathrm{T} 1-\mathrm{T} 2$ & 15 & 77.6 & & & & \\
\hline T3-T4 & 155 & 15.7 & $<0.001$ & 5.267 & $(1.878-14.770)$ & 0.002 \\
\hline \multicolumn{7}{|l|}{$\mathrm{N}$ stage } \\
\hline N0-N1 & 77 & 47.8 & & & & \\
\hline $\mathrm{N} 2-\mathrm{N} 3$ & 93 & 12.7 & $<0.001$ & 1.731 & $(1.036-2.895)$ & 0.036 \\
\hline \multicolumn{7}{|l|}{ Pathological stage } \\
\hline I-II & 53 & 72.5 & & & & \\
\hline III & 117 & 14.1 & $<0.001$ & 8.386 & $(4.415-15.929)$ & 0.000 \\
\hline \multicolumn{7}{|l|}{ NLR } \\
\hline$<2.464$ & 101 & 20.6 & & & & \\
\hline$\geq 2.464$ & 69 & 14.6 & 0.005 & - & - & - \\
\hline \multicolumn{7}{|l|}{ LMR } \\
\hline$<3.279$ & 72 & 15.15 & & & & \\
\hline$\geq 3.279$ & 98 & 21.05 & 0.003 & - & - & - \\
\hline \multicolumn{7}{|l|}{ PNI } \\
\hline$<46.03$ & 102 & 14.8 & & & & \\
\hline$\geq 46.03$ & 68 & 27.5 & $<0.001$ & 1.513 & $(1.015-2.256)$ & 0.042 \\
\hline
\end{tabular}

${ }^{a}$ Adjusted for the following variables: Tumor location, tumor size, $\mathrm{T}$ stage, $\mathrm{N}$ stage, pathological stage and PNI. PNI, prognostic nutritional index; NLR, neutrophil-to-lymphocyte ratio; LMR, lymphocyte-to-monocyte ratio; MST, median survival time; CI, confidence interval.

Subsistence analysis. The average follow-up time for the entire group was 31.7 months (4.6-107.5) months. The postoperative survival rate of patients with PNI $<46.03$ was significantly lower than that of patients with $\mathrm{PNI} \geq 46.03$ (23.7 months vs. 34.3 months, log-rank $\left.\chi^{2}=19.700, \mathrm{P}<0.001\right)$ (Fig. 2).

Univariate analysis and multivariate analysis of postoperative survival in gastric cancer. Univariate analysis showed that tumor location, tumor size, $\mathrm{T}$ stage, $\mathrm{N}$ stage, pathological stage, the NLR, the LMR, and the PNI may be risk factors for survival after $\mathrm{GC}$ radical resection $(\mathrm{P}<0.01)$. The significant results obtained by univariate analysis were included in the Cox regression model. Finally, T3-T4 stage $(\mathrm{HR}=5.267 ; 95 \% \mathrm{CI}$, 1.878-14.770), N2-N3 stage ( $\mathrm{HR}=1.731 ; 95 \% \mathrm{CI}, 1.036-2.895)$, pathological grade III $(\mathrm{HR}=8.386 ; 95 \% \mathrm{CI}, 4.415-15.929)$ and $\mathrm{PNI} \geq 46.03(\mathrm{HR}=1.513$; 95\% CI, 1.015-2.256) were identified as 


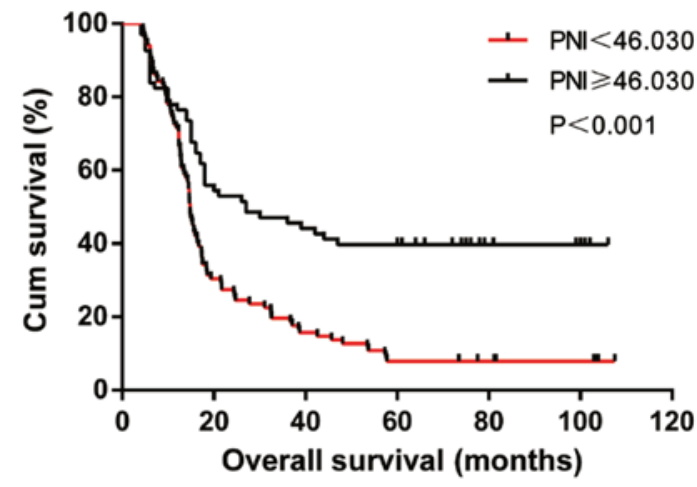

Figure 2. Prognostic K-M curve of PNI and gastric cancer. The postoperative survival rate of patients with PNI $<46.03$ was significantly lower than that of patients with $\mathrm{PNI} \geq 46.03$. The difference between the two groups is statistically significant. PNI <46.030-(102) and PNI $\geq 46.030$-(68). PNI, prognostic nutritional index.

independent risk factors affecting the survival of GC patients after radical surgery (Table III).

\section{Discussion}

GC has been attracting attention in the academic community due to its high morbidity and mortality rates (1). Although surgery has improved the 5 year survival rate of patients with GC, the prognosis is still not optimistic. Gullo et al (15) showed that the prognosis of GC as a highly heterogeneous tumor is closely related to many factors. In addition to the traditional TNM staging system, it is also closely related to the body's immunity, inflammation, and nutrition.

Most patients with GC suffer from nausea, vomiting, abdominal pain, abdominal distension and other symptoms that affect the appetite and diet of the patient. On the other hand, the rapid growth of malignant tumor cells causes the body to consume a large amount of nutrients, resulting in the lack of the synthesis of nutrients, which causes tumor necrosis and the production of toxic substances, leading to metabolic disorders in the body. Therefore, most patients often have different degrees of malnutrition at the time of treatment $(16,17)$. Malnutrition can lead to the loss of optimal treatment timing for patients and the need to delay treatment, which can lead to disease progression. In addition, malnutrition leads to decreased $\mathrm{T}$ cell function and the deterioration of the intestinal environment, further aggravating the progression of tumor-associated inflammation (18). In 1863, since Virchow first proposed the association between inflammation and cancer, the role of inflammation in tumorigenesis, tumor development and metastasis has been continuously explored (19). Mantovani et al (20) showed that tumor-associated inflammation is characterized by the swelling of inflammatory cells and the production and release of inflammatory factors in tumor tissues. Inflammatory cells and immune cells, such as neutrophils, lymphocytes, and monocytes, in the peripheral blood of patients with tumor-associated inflammation are considered to be important factors leading to tumor development, invasion and metastasis (21-24). Existing studies have shown that the NLR and LMR play important roles in determining the prognosis of cancer patients (25-27).
The PNI was first established by Japanese scholars and was originally used to assess the preoperative nutritional status, surgical risk and postoperative complications in surgical patients (12). The PNI is calculated as an indicator of the nutritional status of the body and is based on the level of serum albumin and the number of lymphocytes (12). As the main component of plasma protein, serum albumin plays an important role in maintaining the colloid osmotic pressure of the body; on the other hand, it can also reflect the nutritional status of the body. Ouyang et al (28) showed that preoperative serum albumin levels are associated with prognosis in patients with GC. The immune response of lymphocytes to the tumor has been gradually applied to the prognostic evaluation of tumor patients in recent years (29). The PNI has been used recently for the prognostic evaluation of various tumors, including colorectal cancer (30), liver cancer (31), and pancreatic cancer (32).

Our results show that the preoperative PNI is superior to traditional inflammatory markers for the evaluation of prognosis after GC radical surgery. This may be because the preoperative PNI (in contrast with the traditional inflammatory indicators: The NLR and LMR) can reflect the nutritional status of the body and the body's inflammatory and immune response. A high preoperative PNI and good OS in GC patients suggest that active perioperative nutritional support for GC patients may be a new method of improving patient outcomes. Multivariate analysis indicated that the $\mathrm{T}$ stage, $\mathrm{N}$ stage, pathological grade and PNI were independent risk factors for survival after radical gastrectomy. These results further show that the preoperative PNI may be superior to traditional inflammatory indicators as a potential indicator of the prognosis of patients after radical gastrectomy.

The limitations of this study are as follows: First, the study was a retrospective analysis, and the sample size is small; large-scale, multicenter, prospective studies should be designed. Second, we were not able to collect data on indicators of systemic infections in our research, such as the level of CRP, so we cannot rule out systemic inflammation or the effect of stress on the conclusions of this paper. Because this study was a retrospective study, we only collected data on the patients' survival, but did not have access to complete and effective data on disease-free survival. Therefore, it was not possible to analyze these important prognostic indicators. Finally, the follow-up time was relatively, and a study with a longer-term follow-up period should be designed. In future studies, we will further address the deficiencies.

\section{Acknowledgements}

Not applicable.

\section{Funding}

The present study was supported by the Key Program on Natural Scientific Research from the Department of Education of Anhui Province, China (grant no. KJ2017A219).

\section{Availability of data and materials}

The datasets used and/or analyzed during the present study are available from the corresponding author on reasonable request. 


\section{Authors' contributions}

ML conceived the present study. LW, YM and TC collected the data. LW and DS analyzed the data. LW, LZ and SG wrote the manuscript. LZ and SG supervised the study, performed the statistical analysis and data interpretation, drafted and critically revised the manuscript for important intellectual content. All authors read and approved the final manuscript.

\section{Ethics approval and consent to participate}

The present study was approved by the Clinical Medical Research Ethics Committee of the First Affiliated Hospital of Bengbu Medical College (Bengbu, China). All patients provided signed informed consent.

\section{Patient consent for publication}

Not applicable.

\section{Competing interests}

The authors declare that they have no competing interests.

\section{References}

1. Bray F, Ferlay J, Soerjomataram I, Siegel RL, Torre LA and Jemal A: Global cancer statistics 2018: GLOBOCAN estimates of incidence and mortality worldwide for 36 cancers in 185 countries. CA Cancer J Clin 68: 394-424, 2018.

2. Lin GT, Chen QY, Zheng CH, Li P, Xie JW, Wang JB, Lin JX, Lu J, Cao LL, Lin M, et al: Lymph node noncompliance affects the long-term prognosis of patients with gastric cancer after laparoscopic total gastrectomy. J Gastrointest Surg 1: 10, 2019.

3. Machlowska J, Pucułek M, Sitarz M, Terlecki P, Maciejewski R and Sitarz R: State of the art for gastric signet ring cell carcinoma: From classification, prognosis, and genomic characteristics to specified treatments. Cancer Manag Res 11: 2151-2161, 2019.

4. Siewert JR, Böttcher K, Stein HJ and Roder JD: Relevant prognostic factors in gastric cancer: Ten-year results of the German gastric cancer study. Ann Surg 228: 449-461, 1998.

5. Braga M, Gianotti L, Vignali A and Di Carlo V: Immunonutrition in gastric cancer surgical patients. Nutrition 14: 831-835, 1998.

6. Shi H, Jiang Y, Cao H, Zhu H, Chen B and Ji W: Nomogram based on systemic immune-inflammation index to predict overall survival in gastric cancer patients. Dis Markers 2018: 1787424, 2018.

7. Fujiya K, Kawamura T, Omae K, Makuuchi R, Irino T, Tokunaga M, Tanizawa Y, Bando E and Terashima M: Impact of malnutrition after gastrectomy for gastric cancer on long-term survival. Ann Surg Oncol 25: 974-983, 2018.

8. Sun J, Wang D, Mei Y, Jin H, Zhu K, Liu X, Zhang Q and Yu J: Value of the prognostic nutritional index in advanced gastric cancer treated with preoperative chemotherapy. J Surg Res 209: 37-44, 2017.

9. Zhou J, Hiki N, Mine S, Kumagai K, Ida S, Jiang X, Nunobe S, Ohashi M, Sano T and Yamaguchi T: Role of prealbumin as a powerful and simple index for predicting postoperative complications after gastric cancer surgery. Ann Surg Oncol 24: 510-517, 2017.

10. Rosania R, Chiapponi C, Malfertheiner P and Venerito M: Nutrition in patients with gastric cancer: An update. Gastrointest Tumors 2: 178-187, 2016.

11. Li JH, Han L, Du TP and Guo MJ: The effect of low-nitrogen and low-calorie parenteral nutrition combined with enteral nutrition on inflammatory cytokines and immune functions in patients with gastric cancer: A double blind placebo trial. Eur Rev Med Pharmacol Sci 19: 1345-1350, 2015.

12. Onodera T, Goseki N and Kosaki G: Prognostic nutritional index in gastrointestinal surgery of malnourished cancer patients. Nihon Geka Gakkai Zasshi 85: 1001-1005, 1984 (In Japanese).
13. Japanese Gastric Cancer Association: Japanese gastric cancer treatment guidelines 2014 (ver. 4). Gastric Cancer 20: 1-19, 2017.

14. In H, Solsky I, Palis B, Langdon-Embry M, Ajani J and Sano T: Validation of the 8th edition of the AJCC TNM staging system for gastric cancer using the national cancer database. Ann Surg Oncol 24: 3683-3691, 2017.

15. Gullo I, Carneiro F, Oliveira C and Almeida GM: Heterogeneity in gastric cancer: From pure morphology to molecular classifications. Pathobiology 85: 50-63, 2018.

16. Fukuda Y, Yamamoto K, Hirao M, Nishikawa K, Maeda S, Haraguchi N, Miyake M, Hama N, Miyamoto A, Ikeda M, et al: Prevalence of malnutrition among gastric cancer patients undergoing gastrectomy and optimal preoperative nutritional support for preventing surgical site infections. Ann Surg Oncol 22 (Suppl 3): S778-S785, 2015.

17. Ryu SW and Kim IH: Comparison of different nutritional assessments in detecting malnutrition among gastric cancer patients. World J Gastroenterol 16: 3310-3317, 2010.

18. Fujiya K and Terashima M: ASO author reflections: Malnutrition after gastrectomy and its impact on survival. Ann Surg Oncol 25: 729-730, 2018.

19. Balkwill $\mathrm{F}$ and Mantovani A: Inflammation and cancer: Back to Virchow. Lancet 357: 539-545, 2001.

20. Mantovani A, Allavena P, Sica A and Balkwill F: Cancer-related inflammation. Nature 454: 436-444, 2008.

21. Labelle M, Begum S and Hynes RO: Direct signaling between platelets and cancer cells induces an epithelial-mesenchymal-like transition and promotes metastasis. Cancer Cell 20: 576-590, 2011.

22. Zhang X, Shi H, Yuan X, Jiang P, Qian H and Xu W: Tumor-derived exosomes induce N2 polarization of neutrophils to promote gastric cancer cell migration. Mol Cancer 17: 146 , 2018.

23. Pylaeva E, Harati MD, Spyra I, Bordbari S, Strachan S, Thakur BK, Höing B, Franklin C, Skokowa J, Welte K, et al: NAMPT signaling is critical for the proangiogenic activity of tumor-associated neutrophils. Int J Cancer 144: 136-149, 2019.

24. Huang C, Li Z, Li N, Li Y, Chang A, Zhao T, Wang X, Wang H, Gao S, Yang S, et al: Interleukin 35 expression correlates with microvessel density in pancreatic ductal adenocarcinoma, recruits monocytes, and promotes growth and angiogenesis of xenograft tumors in mice. Gastroenterology 154: 675-688, 2018.

25. Inoue D, Sekiguchi S, Yamagata W, Maeda G, Yamada D, Fujiwara S, Itou S, Kurihara M, Hijioka Y, Shimoji K, et al: Elevation of neutrophil-to-lymphocyte ratio before first-line chemotherapy predicts a poor prognosis for second-line chemotherapy in gastric cancer. Oncology 96: 140-146, 2019.

26. Szor DJ, Roncon Dias A,Pereira MA, Ramos MFKP,Zilberstein B, Cecconello I and Ribeiro U Jr: Neutrophil-Lymphocyte ratio is associated with prognosis in patients who underwent potentially curative resection for gastric cancer. J Surg Oncol 117: 851-857, 2018.

27. Pan YC, Jia ZF, Cao DH, Wu YH, Jiang J, Wen SM, Zhao D, Zhang SL and Cao XY: Preoperative lymphocyte-to-monocyte ratio (LMR) could independently predict overall survival of resectable gastric cancer patients. Medicine (Baltimore) 97: e13896, 2018.

28. Ouyang X, Dang Y, Zhang F and Huang Q: Low serum albumin correlates with poor survival in gastric cancer patients. Clin Lab 64: 239-245, 2018.

29. Rho SY, Hwang HK, Chong JU, Yoon DS, Lee WJ and Kang CM: Association of preoperative total lymphocyte count with prognosis in resected left-sided pancreatic cancer. ANZ J Surg 89: 503-508, 2019.

30. Noh GT, Han J, Cho MS, Hur H, Min BS, Lee KY and Kim NK: Impact of the prognostic nutritional index on the recovery and long-term oncologic outcome of patients with colorectal cancer. J Cancer Res Clin Oncol 143: 1235-1242, 2017.

31. Wang Z, Wang $\mathrm{J}$ and Wang $\mathrm{P}$ : The prognostic value of prognostic nutritional index in hepatocellular carcinoma patients: A meta-analysis of observational studies. PLoS One 13: e0202987, 2018.

32. Li S, Tian G, Chen Z, Zhuang Y and Li G: Prognostic role of the prognostic nutritional index in pancreatic cancer: A meta-analysis. Nutr Cancer 71: 207-213, 2019.

This work is licensed under a Creative Commons Attribution-NonCommercial-NoDerivatives 4.0 International (CC BY-NC-ND 4.0) License. 